\title{
Revisiting the Concept of Non- and Minimally Invasive Interventions in Early Glottic Cancer - Part II: Single Therapy Should be Favored over the Combination of Transoral Laser Microsurgery and Radiotherapy, Regarding the Postinterventional Voice Quality
}

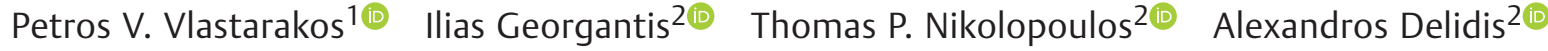 \\ 1 Department of ENT, MITERA Hospital, Athens, Greece \\ 2 Department of ENT, Attikon University Hospital, Athens, Greece \\ Address for correspondence Petros V. Vlastarakos, MD, MSc, PhD, \\ IDO-HNS (Eng.), 58 Laskaridou Str., Kallithea-Athens 17676, Greece \\ (e-mail: pevlast@hotmail.com; pevlast@yahoo.gr).
} Int Arch Otorhinolaryngol 2022;26(3):e310-e313.

\begin{abstract}
Introduction Early glottic cancer (EGC) is associated with a high cure rate. Hence, patients and physicians also focus on the impact of the proposed treatment on the speaking function of the preserved larynx.

Objectives The present study assessed the impact of single-modality treatment (transoral laser microsurgery [TLM], or radiotherapy) or combination therapy for EGC on postinterventional voicing and explored factors which might explain the related perceptions. Methods A total of 108 patients filled in the voice handicap index 10 questionnaire, 1 and 2 years postinterventionally. Non-parametric tests were used for the respective statistical analyses.

Results Sixty-four patients were treated with TLM, 15 with radiotherapy, and 29 with both modalities. Transoral microsurgery and radiotherapy were associated with postinterventional dysphonia, which attenuated between the first and second postintervention year $(p=0.000)$. No association between sociodemographic parameters and the attenuation of postinterventional dysphonia was identified for either treatment modality. Transoral microsurgery and radiotherapy resulted in comparable postinterventional voicing, in the first $(p=0.940)$ and second $(p=0.196)$ postintervention years. The addition of TLM to radiotherapy resulted in worse voice quality in the second, compared with the first postintervention year $(p=0.000)$, demonstrating a detrimental effect on speech intelligibility in noise $(p=0.000)$.
Keywords
Conclusion Single therapy should be favored over the combination of TLM and
- cancer radiotherapy for EGC in terms of retaining better postinterventional voice quality.
- glottis
- dysphonia
- laser
- radiotherapy Postinterventional dysphonia should be taken into account, during preinterventional counseling, as it may exert leverage on the quality of patients' lives. Patients and physicians should acknowledge the optimal time of voice function return, which seems to be extending up to two years posttreatment.

received

January 22, 2020

accepted

February 23, 2021

published online

August 13, 2021
DOI https://doi.org/ 10.1055/s-0041-1730454. ISSN 1809-9777.

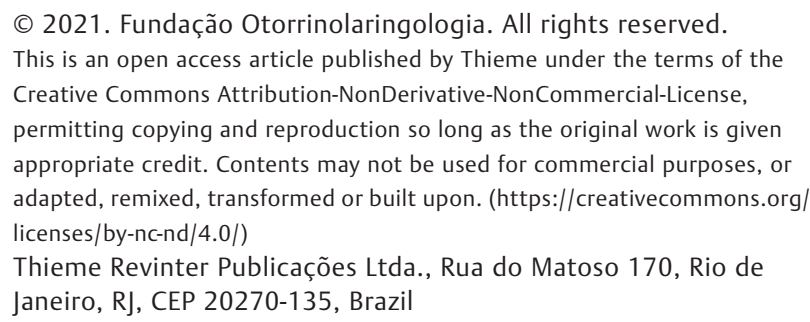

(c) 2021. Fundação Otorrinolaringologia. All rights reserved. This is an open access article published by Thieme under the terms of the Creative Commons Attribution-NonDerivative-NonCommercial-License, permitting copying and reproduction so long as the original work is given appropriate credit. Contents may not be used for commercial purposes, or adapted, remixed, transformed or built upon. (https://creativecommons.org/ licenses/by-nc-nd/4.0/) Thieme Revinter Publicações Ltda., Rua do Matoso 170, Rio de Janeiro, RJ, CEP 20270-135, Brazil 


\section{Introduction}

Laryngeal cancer is one of the most common head and neck cancers, with glottic carcinoma representing the majority of cases. ${ }^{1}$ Early detection and treatment of laryngeal cancer can lead to a cure rate ranging from 80 to $90 \%{ }^{2}$ With a survival rate of this magnitude, it is not unreasonable for patients and ENT surgeons to focus not only on the disease, but also on the impact of the proposed treatment on the speaking function of the preserved larynx, to provide realistic patient expectations. $^{3}$

Hence, clinical research over the past 25 years has focused on efforts to preserve the laryngeal function, through improvements in single-modality approach of patients with early glottic cancer. ${ }^{2}$ Such treatment options include larynx-preserving surgery (transoral laser microsurgery [TLM]) and irradiation (or their combination, when deemed necessary).

The aim of the present study was to assess the impact of single-modality treatment (TLM or radiotherapy, respectively), or combination therapy for early glottic cancer (EGC) on postinterventional voicing as well as to identify factors which might explain the related perceptions. Sociodemographic parameters potentially associated with postinterventional voicing in patients with EGC were also explored.

\section{Patients \& Methods}

A prospective study was conducted at a tertiary university hospital, involving 108 patients who had been treated for EGC between 2012 and 2016. The patients had been subjected either to TLM $(n=64)$ or external irradiation $(n=15)$ or had received both treatment modalities in combination $(n=29)$. The inclusion criteria involved: a) stage I or II of glottic cancer, $\mathrm{b})$ age $\leq 78$ years. This parameter ensured that patients would not be older than 80 years old at the second follow-up appointment, thus minimizing age-related voice disorders, c) absence of heart failure, chronic obstructive pulmonary disease, psychiatric, or neurodegenerative disorders, and d) competency in reading, writing, and understanding the Greek language.

The patients filled in the voice handicap index 10 (VHI10), ${ }^{4}$ which is a validated robust short version of the VHI used to quantify the patients' perceptions of their voice disorders in relation to their actual severity, ${ }^{5}$ both for screening and interpretation purposes. The questionnaires were completed 1 and 2 years after the respective intervention. The VHI-10 consists of 10 items, which assess functional, physical, and emotional domains of voice disorders, in relation to the postinterventional status of patients treated for EGC. ${ }^{4}$ There are five possible responses to each item (never, almost never, sometimes, nearly always, and always). Individual scores for each item range from 0 to 4 , and the total score from 0 to 40 . Higher questionnaire scores reflect worse postinterventional voicing and voicing-related quality of life. The questionnaire has been appropriately validated for the Greek language (GVHI-10). ${ }^{6}$ Completion time typically lasted around 15 minutes.
Preliminary statistic control of group data, both with the Kolmogorov-Smirnov and the Shapiro-Wilk tests, was not suggestive of satisfying fitting to the normal distribution; hence, non-parametric tests were used to perform the respective statistical analyses. The Mann-Whitney test was used to compare the total VHI-10 scores, between the studied groups, in the first and second post-intervention year. The Wilcoxon signed rank test compared the total VHI-10 scores within the studied groups, between the $1^{\text {st }}$ and $2^{\text {nd }}$ postintervention years, and boxplots depicted the observed differences. The potential association between sociodemographic parameters and postinterventional voicing was assessed using the Kruskal-Wallis test for occupation, marital, and educational status, the MannWhitney test for gender, and the Spearman correlation coefficient for age. All analyses were performed using the SPSS Statistics for Windows, Version 25.0 (IBM Corp., Armonk, NY, USA). Statistical significance was accepted at the level of 0.05 .

The research protocol was submitted, and ethical approval was received by the ethics committee of the university to which the Academic Hospital is affiliated, prior to commencing data collection. The participants were asked to sign a consent form before being enrolled in the study.

\section{Results}

Among the 108 patients who participated in the study, 88 were male and 20 were female. The mean age was 61.95 ( \pm 9.27 ) years, ranging between 45 and 78 years of age (in accordance with the inclusion criteria).

Transoral laser microsurgery was associated with postinterventional dysphonia (VHI score $13.89 \pm 3.93$ ), which, however, attenuated between the $1^{\text {st }}$ and $2^{\text {nd }}$ postintervention years (VHI score $12.50 \pm 4.06)(p=0.000)$ (-Fig. 1). Similar results were obtained in irradiated patients, in whom the occurrence of postinterventional dysphonia (VHI score $13.73 \pm 1.79$ ) mitigated in the $2^{\text {nd }}$ postintervention year (VHI score $10.80 \pm 1.78)(p=0.000)$ (-Fig. 2). In addition, TLM and radiotherapy demonstrated comparable postinterventional voicing, in the $1^{\text {st }}(p=0.940)$ and $2^{\text {nd }}$ $(p=0.196)$ postintervention years.

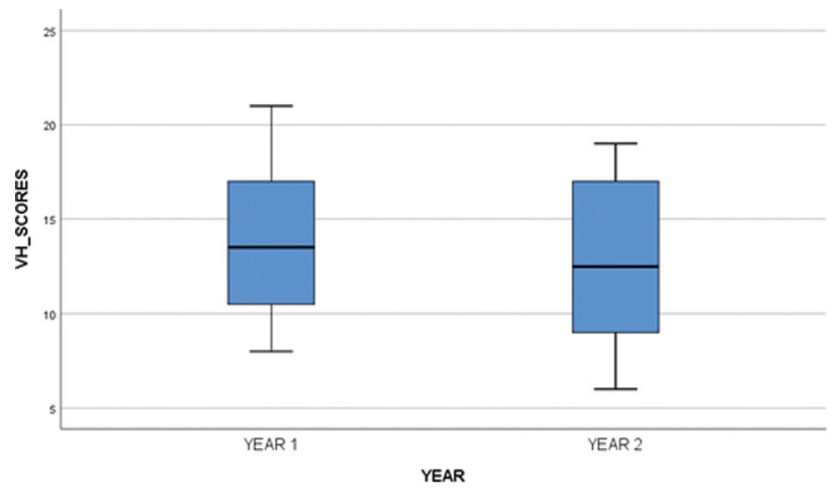

Fig. 1 Boxplot depicting the attenuation of dysphonia between the first and second postinterventional year in patients treated with transoral laser microsurgery. 


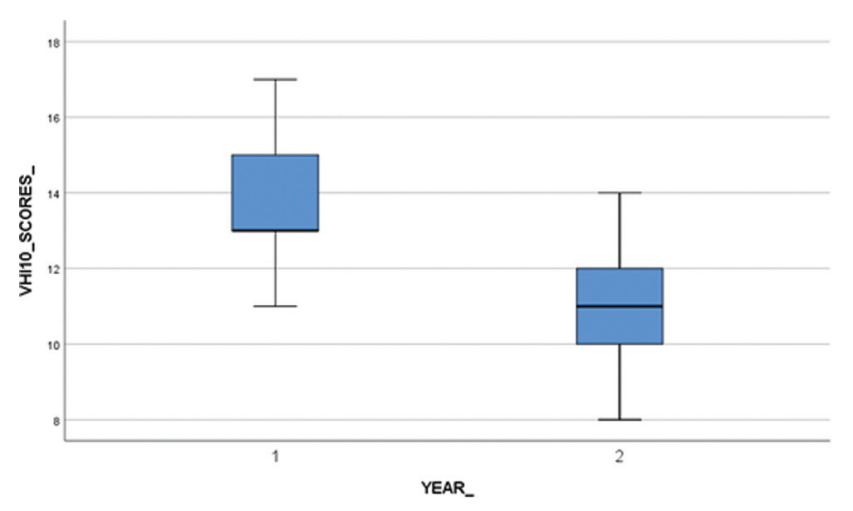

Fig. 2 Boxplot depicting the attenuation of dysphonia between the first and second postinterventional year in patients treated with external irradiation.

No association between sociodemographic parameters and the attenuation of postinterventional dysphonia was identified for either treatment modality. The respective analyses included age $\left(\mathrm{p}_{\mathrm{TLM}}=0.785, \mathrm{p}_{\mathrm{R}}=0.109\right)$, gender $\left(\mathrm{p}_{\mathrm{TLM}}=0.178, \mathrm{p}_{\mathrm{R}}=0.819\right)$, occupation $\left(\mathrm{p}_{\mathrm{TLM}}=0.808, \mathrm{p}_{\mathrm{R}}\right.$ $=0.477)$, marital status $\left(\mathrm{p}_{\mathrm{TLM}}=0.823, \mathrm{p}_{\mathrm{R}}=0.235\right)$, and education $\left(\mathrm{p}_{\mathrm{TLM}}=0.322, \mathrm{p}_{\mathrm{R}}=0.497\right)$.

By contrast, when patients treated with TLM were compared with those treated with additional radiotherapy, they demonstrated worse voice quality in the $1^{\text {st }}(p=0.010)$ and $2^{\text {nd }}(p=0.048)$ postintervention years. Furthermore, the comparison between patients treated with radiotherapy and those treated with radiotherapy additional to TLM resulted in worse voice quality in the $2^{\text {nd }}$, compared with the $1^{\text {st }}$ postintervention year $(p=0.000)$. Such patients were demonstrating a detrimental effect on speech intelligibility in noise $(p=0.000)$ in the $2^{\text {nd }}$, compared with the $1^{\text {st }}$ postintervention year $(p=0.000)$.

\section{Discussion}

Appropriate treatment planning for EGC involves an initial selection between TLM and external irradiation. However, a key characteristic of both the disease and the proposed treatment is the related impact on voice quality. Bearing in mind that the primary goal of any treatment strategy for EGC is securing local disease control, a secondary aim would be to provide the best possible quality of postinterventional voicing. Nevertheless, patients treated for EGC need to have realistic expectations, accepting the fact that postinterventional dysphonia will occur, despite the minuscule proposed intervention, and regardless of the utilized treatment modality. That is because TLM results in loss of vocal cord tissue, while radiotherapy leads to vocal fold fibrosis and muscle fatigue. ${ }^{7}$ However, the ensuing dysphonia is expected to attenuate between the $1^{\text {st }}$ and $2^{\text {nd }}$ postintervention years, following either treatment modality.

Single-modality therapy, as opposed to the combination of TLM and radiotherapy, is favored for patients suffering from EGC, regardless of the utilized treatment modality, ${ }^{2}$ in terms of retaining better postintervention voice quality, provided, of course, that no medical reason dictates their combined use. This approach is confirmed by the results of the present study, following a two-angled comparison strategy. Indeed, when TLM patients $(n=64)$ were compared with their TLM and radiotherapy counterparts $(n=29)$, the latter group demonstrated worse voice quality in both the $1^{\text {st }}$ $(p=0.010)$ and the $2^{\text {nd }}(p=0.048)$ postintervention years. Furthermore, when radiotherapy patients $(n=15)$ were compared with their TLM and radiotherapy counterparts $(n=29)$, the latter group demonstrated a deteriorating voice quality in the $2^{\text {nd }}$ postintervention year, in comparison with the respective outcomes of the $1^{\text {st }}$ year after treatment $(p=0.000)$.

While the improvement of postoperative VHI-10 scores in patients with EGC treated with TLM is well established and seems, in fact, to be extending well into the $2^{\text {nd }}$ postoperative year, ${ }^{8}$ meta-analyses have indicated that the perceptive and acoustic vocal quality of their radiotherapy-only counterparts may be even better. ${ }^{1,9}$ However, by applying element analysis of the VHI-10, the present study identified that when these radical single-treatment modalities are combined, the postinterventional intelligibility of the patients' speech in noisy environments may pose as the primary parameter of voice handicap in the $2^{\text {nd }}$ postintervention year $(p=0.000)$.

By contrast, the progress of postinterventional dysphonia in early glottic cancer patients treated with single-modality approach, does not seem to be associated with sociodemographic parameters, including age, gender, occupation, marital, or educational status. This finding is quite important, taking the rising rates of laryngeal cancer in women into account, ${ }^{10}$ along with the population aging in Western societies, as socio-demographic parameters related to occupation and marital status have been associated with laryngeal cancer incidence per se, ${ }^{11}$ while advanced age has been considered an independent predictor of worse overall (hazard ratio $=1.4$ ), and cancer-specific (hazard ratio $=1.2$ ) survival in head and neck patients, even in the presence of an otherwise fairly equal access to care. ${ }^{12}$ Furthermore, a higher socio-economic level might lead to more timely perception of symptom importance, earlier access to health and dental services, and thus diagnosis of head and neck cancer at an earlier stage. ${ }^{13}$

On the other hand, quality of life considerations arising either from the disease, and/or from the proposed treatment, need to be analyzed during pre-interventional counseling, as they may be linked to socio-demographic factors, and exert leverage on different aspects of patients' lives (i.e., social life, psychological well-being, financial implications). The attenuation of post-interventional dysphonia in early glottic cancer should be viewed in this context, taking into account the importance of identifying voice outcomes as a function of time on patients'quality of life. Indeed, patients and physicians should realize the non-linear course of postinterventional improvement in voice handicap, ${ }^{8}$ and acknowledge the optimal time of voice function return, which does seem to be extending up to two years posttreatment, according to the results of the present study.

Strengths of the present study include the use of a validated questionnaire, with a completion time not 
exceeding 15 minutes, along with a robust statistical analysis. Limitations of the present study include its single-center setting, along with a slight underestimation of the mean age of patients treated for early glottic cancer. The former limitation is counterbalanced by the fact that the study took place at a tertiary academic hospital, which accepts referrals at a nationwide level. The latter limitation was inextricably related to setting the upper age limit for patient selection to 78 years. However, this age-related parameter ensured that patients would not be older than 80 years old at the second follow up appointment, thus minimizing the potential impact of age-related voice disorders.

\section{Conclusion}

Single-modality therapy, as opposed to the combination of TLM and radiotherapy, is favored for patients suffering from EGC, regardless of the utilized treatment modality, in terms of retaining better postinterventional voice quality. Postinterventional dysphonia occurs, regardless of sociodemographic parameters, in patients treated for EGC and should be taken into account during preinterventional counseling, as it may exert leverage on the quality of patients' lives. Patients and physicians should realize the non-linear course of postintervention improvement in voice handicap and acknowledge the optimal time of voice function return, which does seem to be extending up to 2 years posttreatment.

\section{Ethical Approval}

All procedures performed were in accordance with the ethical standards of the institutional and national research committee and with the 1964 Helsinki declaration and its later amendments.

The research protocol was submitted and received ethical approval by the Ethics Committee of the University of Athens, prior to commencing data collection. Participants were asked to sign a consent form before being enrolled in the study.

\section{Funding}

The authors have no financial interest and have not received any financial support for this article.

\section{Conflict of Interests}

The authors declare that there is no conflict of interests.

\section{References}

1 Lee SH, Hong KH, Kim JS, Hong YT. Perceptual and Acoustic Outcomes of Early-Stage Glottic Cancer After Laser Surgery or Radiotherapy: A Meta-Analysis. Clin Exp Otorhinolaryngol 2019; 12(03):241-248

2 Forastiere AA, Ismaila N, Lewin JS, et al. Use of Larynx-Preservation Strategies in the Treatment of Laryngeal Cancer: American Society of Clinical Oncology Clinical Practice Guideline Update. J Clin Oncol 2018;36(11):1143-1169

3 Starmer HM, Tippett DC, Webster KT. Effects of laryngeal cancer on voice and swallowing. Otolaryngol Clin North Am 2008;41 (04):793-818, vii

4 Rosen CA, Lee AS, Osborne J, Zullo T, Murry T. Development and validation of the voice handicap index-10. Laryngoscope 2004; 114(09):1549-1556

5 Behlau M, Zambon F, Moreti F, Oliveira G, de Barros Couto E Jr. Voice self-assessment protocols: different trends among organic and behavioral dysphonias. J Voice 2017;31(01):112.e13-112.e27

6 Helidoni ME, Murry T, Moschandreas J, Lionis C, Printza A, Velegrakis GA. Cross-cultural adaptation and validation of the voice handicap index into Greek. J Voice 2010;24(02):221-227

7 Hong YT, Park MJ, Hong KH. Characteristics of speech production in patients with T1 glottic cancer who underwent laser cordectomy or radiotherapy. Logoped Phoniatr Vocol 2018;43(03):120-128

8 Lane C, Rigby M, Hart R, Trites J, Levi E, Taylor SM. Longitudinal analysis of Voice Handicap Index in early glottic cancer patients treated with transoral laser microsurgery: age, gender, stage and time dependence. J Laryngol Otol 2019;133(04):318-323

9 Guimarães AV, Dedivitis RA, Matos LL, Aires FT, Cernea CR. Comparison between transoral laser surgery and radiotherapy in the treatment of early glottic cancer: A systematic review and meta-analysis. Sci Rep 2018;8(01):11900

10 Kachuri L, De P, Ellison LF, Semenciw RAdvisory Committee on Canadian Cancer Statistics. Cancer incidence, mortality and survival trends in Canada, 1970-2007. Chronic Dis Inj Can 2013;33 (02):69-80

11 Guenel P, Engholm G, Lynge E. Laryngeal cancer in Denmark: a nationwide longitudinal study based on register linkage data. $\mathrm{Br} \mathrm{J}$ Ind Med 1990;47(07):473-479

12 Choi SH, Terrell JE, Fowler KE, et al. Socioeconomic and Other Demographic Disparities Predicting Survival among Head and Neck Cancer Patients. PLoS One 2016;11(03):e0149886

13 Vlastarakos PV, Gkouvali A, Katsochi D. Attitudes and Parameters Affecting the Behavior Toward Precursor Symptoms of Head and Neck Cancer. Ear Nose Throat J 2019;98(06):E58-E63 